allele $(p=0.03)$ compared to FDRs without the ${ }^{*} 0405$ allele. Being positive for the SE was not significantly associated with sICAM, sVCAM, or E-selectin levels. Conclusions: In RA-free FDRs, having an HLA-DRB1 ${ }^{*} 0404$ or HLA-DRB1*0405 allele was associated with markers of endothelial injury. Therefore, the genetic predisposition to RA could contribute to parallel development of atherosclerosis during the preclinical period of RA.

References:

[1] Gonzalez-Juanatey C et al., Am J Med 2003; 114:647-52.

[2] Gonzalez-Gay MA et al., Arth Rheum 2007; 57(1):125-132.

[3] Gersuk VH et al., J Immunol Methods. 2006 Dec 20; 317(1-2): 64-70.

Disclosure of Interest: None declared

DOI: 10.1136/annrheumdis-2017-eular.4570

\section{FRI0707 DRUG LEVELS AND ANTIDRUG ANTIBODIES IN THE DEVELOPMENT OF PARADOXICAL PSORIASIS AND PALMOPLANTAR PUSTULOSIS}

J. Ruwaard $^{1}$, E. van der Vlugt ${ }^{1}$, P. Brevet ${ }^{1}$, L. Berkhout ${ }^{2}$, T. Rispens ${ }^{2}$, M. Nurmohamed ${ }^{1}$, G.J. Wolbink ${ }^{1,2} .{ }^{1}$ Amsterdam Rheumatology and immunology Center / Reade; ${ }^{2}$ Department of Immunopathology, Sanquin Research and Landsteiner Laboratory Academic Medical Centre, Amsterdam, Netherlands

Background: The pathogenesis of psoriasis and palmoplantar pustulosis induced by Tumor Necrosis Factor inhibitors (TNFi) is largely unknown. Only one study, in 9 inflammatory bowel disease patients, investigated the relation with infliximab drug levels in the development of psoriasis or palmoplantar pustulosis and demonstrated no relation with of trough concentrations in these events (1). However, psoriasis and palmoplantar pustulosis were not studied separately. Objectives: To study the differences in drug levels and antidrug antibodies (ADA) of TNFi in rheumatoid arthritis (RA) and ankylosing spondylitis (AS) patients who developed de novo psoriasis, palmoplantar pustulosis and those who did not develop skin adverse events.

Methods: In this retrospective study data was collected from the observational cohorts of Reade of consecutive RA and AS patients in whom TNFi was started. At every visit, serum samples were collected. We quantified the samples before/on time of event of the patients who developed psoriasis or palmoplantar pustulosis, for the patients with no skin adverse events (control group) at 24 or 28 weeks. Drug levels and ADA were measured with an Enzyme-linked immunosorbent assay and antibody binding test respectively.

Results: A total of 830 TNFi naive patients with RA and AS were included, of whom 21 developed psoriasis $(n=11)$ or palmoplantar pustulosis $(n=10)$. These patients were only observed in the adalimumab and etanercept cohorts. Sixteen patients with an event and 585 patients in the control group had serum samples available to quantify drug levels and ADA. No statistical significant differences were found in drug levels of adalimumab and etanercept for both RA and AS patients (table 1). Moreover, no statistical significant differences were observed in the detection of ADA between the three groups. However, no ADA were detected in patients who developed psoriasis or palmoplantar pustulosis compared to the overall $13.9 \%$ of the RA patients and $25.5 \%$ in AS patients.

Table 1. Differences in drug levels and detection of anti-drug antibodies between palmoplantar pustulosis, psoriasis and control group

\begin{tabular}{|c|c|c|c|c|c|c|}
\hline \multirow[b]{2}{*}{ RA } & \multicolumn{2}{|c|}{$\begin{array}{c}\text { Druglevels } \\
\text { adalimumab } \\
(\mu \mathrm{g} / \mathrm{ml} ; \text { median (IQR) }\end{array}$} & \multicolumn{2}{|c|}{$\begin{array}{c}\text { Drug levels } \\
\text { etanercept } \\
(\mu \mathrm{g} / \mathrm{ml}) ; \text { median }(\mathrm{IQR})\end{array}$} & \multicolumn{2}{|c|}{$\begin{array}{c}\text { Anti-drug } \\
\text { antibodies } \\
\text { adalimumab, } \\
\text { no. (\%) }\end{array}$} \\
\hline & & & & & & \\
\hline Palmoplantar pustulosis & $\mathrm{n}=3$ & $7,6(0,01-12,0)$ & $\mathrm{n}=1$ & 4,4 & $\mathrm{n}=3$ & $0(0)$ \\
\hline Psoriasis & $\mathrm{n}=3$ & $8,5(6,5-10,0)$ & $\mathrm{n}=2$ & $2,3(1,5-3,1)$ & $\mathrm{n}=2$ & $0(0)$ \\
\hline Control group & $n=153$ & $7,4(4,0-10,0)$ & $\mathrm{n}=89$ & $2.7(1.9-3.9)$ & $\mathrm{n}=151$ & $21(13,9)$ \\
\hline$p$-value & & 0,992 & & 0,380 & & 674 \\
\hline \multicolumn{7}{|l|}{ AS } \\
\hline Palmoplantar pustulosis & $\mathrm{n}=3$ & $9,0(6,5-10,0)$ & $\mathrm{n}=1$ & 1,7 & $n=3$ & $0(0)$ \\
\hline Psoriasis & $\mathrm{n}=1$ & 10,0 & $\mathrm{n}=2$ & $1,3(0,8-1,7)$ & $\mathrm{n}=1$ & $0(0)$ \\
\hline Control group & $\mathrm{n}=46$ & $8,5(3,7-11,3)$ & $\mathrm{n}=99$ & $2.6(1.4-4.0)$ & $\mathrm{n}=47$ & $12(25,5)$ \\
\hline p-value & & 0,754 & & 0,406 & & 754 \\
\hline
\end{tabular}

RA: rheumatoid arthritis; AS: ankylosing spondylitis; Control group: patients who did not develop skin adverse events; IQR: interquartile range; no. number of patients. p-value $<0,05$ was considered statistically significant.

Conclusions: Patients who develop paradoxical psoriasis and palmoplantar pustulosis have adequate drug levels and no ADA were detected.

References:

[1] J Crohns Colitis 2015 Nov;9(11):982-7.

Disclosure of Interest: None declared

DOI: 10.1136/annrheumdis-2017-eular.3635

\section{FRI0708 NEUROPATHIC PAIN IS A WEAK PREDICTOR OF NEW ONSET CHRONIC WIDESPREAD PAIN}

J. McBeth ${ }^{1}$, M. Mulvey ${ }^{2}$, A. Rashid ${ }^{1}$, J. Anderson ${ }^{1}$, K. Druce ${ }^{1} .{ }^{1}$ Arthritis Research UK Centre for Epidemiology, The University of Manchester, Manchester; ${ }^{2}$ Leeds University, Leeds, United Kingdom

Background: Regional pain (e.g. back pain) predicts incident chronic widespread pain (CWP), the clinical hallmark of fibromyalgia. Up to $20 \%$ of patients with CWP have neuropathic pain (NP). People with CWP and NP report similar pain characteristics including allodynia (pain in response to normal touch), have common risk factors (age, sex, body mass index, smoking and socioeconomic status) and a shared genetic predisposition. Whether NP is a risk factor for CWP is not known.

Objectives: To test the hypothesis that among persons free of CWP, NP would increase the risk of developing CWP.

Methods: In a population based study participant's pain reports were coded and those free of CWP (ACR criteria: pain lasting $>3$ months in the axial skeleton and contralateral body quadrants) identified. Participants also completed the Douleur Neuropathique 4 (DN4) (which has 7 sensory descriptors of pain (burning, painful cold, electric shocks, tingling, pins and needles, itching, and numbness), scores $\geq 3$ indicating NP); demographics (date of birth, sex, English Index of Multiple Deprivation, occupational status); Hospital Anxiety and Depression (HAD) scale; Estimation of Sleep Problem Scale (ESPS); self-reported pain medications (summed to give a total count); and signed consent. Participants were classified as no pain, having some pain that wasn't neuropathic (NP-; DN4 score $<3$ ), or neuropathic pain (NP+; DN4 score $\geq 3$ ). A follow-up questionnaire mailed 12 months later gathered pain data using the methods in the baseline survey. Based upon their pain reports at follow up participants were classified as "new CWP" for those who reported pain that satisfied the criteria for CWP, or "not CWP". Logistic regression estimated the odds of developing new CWP in the NP-, and $\mathrm{NP}+$ groups compared to the no pain group. Results were expressed as odds ratios (OR) with $95 \%$ confidence intervals $(\mathrm{Cl})$. Population attributable fractions (PAF) estimated the \% of new CWP that would be avoided if participants were not exposed to NP- or NP+.

Results: A total of 1162 participants who were free of CWP, completed the DN4 and provided pain data at follow up. Of those $523(45.0 \%)$ had no pain at baseline, $562(48.4 \%)$ had NP- and $77(6.6 \%)$ had NP+. New onset CWP was reported by $153(13.2 \%)$ participants; $19(3.6 \%)$ of the no pain group, $108(19.2 \%)$ of the NP- group, and $26(33.8 \%)$ of the NP+ group. After adjusting for age and sex, compared to the no pain group, the NP- group was 3 times (OR 2.9, 95\% Cl $(2.0$, $4.2))$ and the NP+ group 4 times $(3.9(2.3,6.4))$ more likely to have new CWP at follow up. These relationships were attenuated but persisted after adjustments for demographics, HAD, ESPS and medication use (NP- $(2.9(1.9,4.3)$; NP+ $(2.1$ $(1.1,4.0))$. The PAF was $41.3 \%(95 \% \mathrm{Cl}(25.2,54.0))$ for NP- and $6.0 \%(0.1$, 11.6) for NP+. All of the individual DN4 characteristics except painful cold and itching predicted new CWP with PAF's ranging from $1.6 \%(0.1,3.8)$ for pins and needles to $5.0 \%(1.1,8.8)$ for burning

Conclusions: NP predicts a small number of new onset CWP cases. CWP is highly prevalent in the general population and effective treatment of pain not of NP origin will have a significant impact on population levels of CWP.

Disclosure of Interest: None declared

DOI: 10.1136/annrheumdis-2017-eular.1642

\section{FRI0709 PREVALENCE OF RHEUMATIC DISEASES BASED ON COPCOPRD STUDIES: A SYSTEMATIC REVIEW}

J. Moreno-Montoya $^{1}$, J. Rodriguez-Amado ${ }^{2}$, R. Burgos-Vargas ${ }^{3}$, I. Pelaez-Ballestas ${ }^{3} .{ }^{1}$ Universidad del Bosque, Bogota, Colombia; ${ }^{2}$ Research Associate of South Florida, Miami, United States; ${ }^{3}$ Rheumatology Unit, Hospital General de Mexico, Mexico, Mexico

Background: Despite many efforts, to date there has been no focused attempt to derive a robust estimate of the prevalence of rheumatic diseases (RDs) to quantify how this is influenced by other factors than them examined in every local study, however, the problems magnitude is rising and due the demographic transition and the increase in the life expectancy

Objectives: To determine, through a systematic review and meta-analysis, the prevalence of RDs in the adult general population and explore its heterogeneity Methods: MEDLINE, EMBASE, BIREME, LILLACS and Web of Science were searched using a search strategy combining key words and related databasespecific subject terms to identify relevant cross-sectional based on COPCORD methodology studies. Also was developed a manual search. Included articles were assessed for risk of bias and quality based on the STROBE statement. Prevalence figures for RDs were analyzed according to female percentage of sampled individuals, mean age and sample size. A mixed effect model was used to obtain the combined prevalence and a meta-regression to estimate the effects of other variables

Results: 44 out from 127 papers were included in English, Spanish or Portuguese. Estimates for any RDs prevalence ranged from $7.2 \%$ to $62.3 \%(26.9 \% ; 95 \% \mathrm{Cl}$ $18.3 \%>25.6 \%$ ). For rheumatoid arthritis (RA), the prevalence varied between $0.2 \%$ and $6.2 \%(1.04 \% ; 95 \% \mathrm{Cl} 0.4 \%>1.6 \%)$; fibromyalgia (FM) had a mean prevalence of $2.1 \%(95 \% \mathrm{Cl} 1.0 \%>3.2 \%)$ and osteoarthritis: $13.5 \%(95 \% \mathrm{Cl}$ : $10.6 \%>16.4 \%$ ). SLE (systematic lupus erythematosus) was the less frequent condition with average prevalence of $0.14 \%(95 \% \mathrm{Cl}: 0.005 \%>0.28 \%)$. The random-effects pooled prevalence for any RDs was $25 \%(95 \% \mathrm{Cl}: 18.0 \%$, $31.1 \%$ ). Prevalence was higher in studies with bigger sample size (random effect coefficient: $0.0014, p=002$ ). There was evidence of relevant heterogeneity in the analysis $(p<0,001)$ and for RDs, RA and FM the sample size was positively associated to the perceived heterogeneity. No effects were found for SLE Conclusions: It was found significant variation among the prevalence across this 


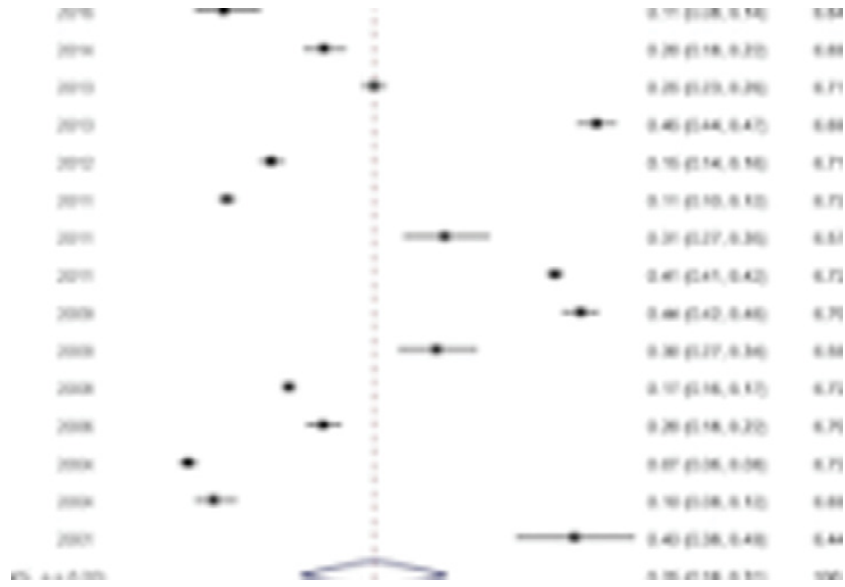

review, in particular, related to the sample size used in each study. Two facts must be accounted for, first the statistical difficulties associated to the estimation of small prevalence and the consequent heterogeneity of the estimates, and, second, the limited number of studies included in this meta-analysis. Nonetheless, there is evidence about big heterogeneity what can correspond to non-observed variables, in particular, life-styles, and environmental or genetic traits.

Disclosure of Interest: None declared

DOI: 10.1136/annrheumdis-2017-eular.3919

\section{FRI0710 MONOSODIUM URATE CRYSTAL DEPOSITION ASSOCIATED WITH THE CHANGE OF RADIOGRAPHIC GRADE AT THE SACROILIAC JOINT IN AXIAL SPA: A DUAL-ENERGY CT STUDY}

J. Zhu $^{1}$, A. Aiwu Li ${ }^{2}$, E. Jia ${ }^{2}$, Y. Zhou ${ }^{3}$, J. Xu$^{2}$, S. Chen ${ }^{2}$, Y. Huang ${ }^{2}$, X. Xiao ${ }^{4}$, J. Li ${ }^{2} .{ }^{1}$ Department of Rheumatology, Nanfang Hospital, Southern Medical University; ${ }^{2}$ Department of Internal Medicine of Traditional Chinese Medicine, College of Traditional Chinese Medicine, Southern Medical University; ${ }^{3}$ Department of Obstetrics, Guangdong Women and Children Hospital; ${ }^{4}$ Department of Radiology, Nanfang Hospital, Southern Medical University, Guangzhou, China

Background: Previous studies have revealed that ankylosing spondylitis (AS), as the progenitor of axial spondyloarthritis (AxSpA), has been characterized by the insidiously progressive nature of sacroiliitis and spondylitis. Dual-energy computed tomography (DECT) has recently been used to analyse the deposition of monosodium urate (MSU) crystals with higher sensitivity and specificity. However, it remains unclear whether the existence of the MSU crystal deposits detected by DECT at the sacroiliac joint in patients with AxSpA also contributed to the existing structural damage.

Objectives: We performed this study to show the DECT MSU crystal deposits in AxSpA patients without coexisting gout and to ascertain whether the MSU crystal deposits at the sacroiliac joint in those patients increased the risk of the structural joint damage.

Methods: One hundred and eighty-six AxSpA patients without coexisting gout were recruited. The plain radiographs of the sacroiliac joint were obtained, along with the DECT scans at the pelvis and the clinical variables. All statistics based on the left or right sacroiliac joint damage grading (0-4) were calculated independently. Bivariate analysis and ordinal logistic regression was performed between the clinical features and radiographic grades at the sacroiliac joint.

Results: At painful joints or skeleton regions, large quantities of MSU crystal deposits were found in 186 patients with $\mathrm{AxSpA}$, as depicted in green with DECT. The average MSU crystal volume at the left sacroiliac joint, the right sacroiliac joint, and the pelvis were $0.902 \pm 1.345,1.074 \pm 1.878$, and $5.272 \pm 9.044$ $\mathrm{cm}^{3}$, values which were correlated with serum uric acid concentrations $(r=0.727$, $0.740,0.896 ; p<0.001)$. At the left and right sacroiliac joint, the presence of MSU crystal deposits $(=11.451,43.684 ; p<0.01)$ and the volumes of MSU crystals ( $Z=9.198, Z=34.607 ; p<0.05)$ were statistically different among groups divided by the ASDAS scores. In bivariate analysis, wide clinical variables were associated with the changes in sacroiliac joint damage. When others factors were adjusted in the ordinal logistic models, the AxSpA duration, total back pain, BASFI score, and volume of MSU crystallization were the risk factors for the radiographic grade at the left sacroiliac joint $(\mathrm{AOR}=1.187,1.428,3.837,2.018 ; p<0.05)$. The same risk factors were obtained for the right sacroiliac joint, except for total back pain. Additionally, the simplified models excluded the repeated variables; the AxSpA duration, BASFI score, and the volume of MSU crystallization at both sides of sacroiliac joint served as risk factors for the radiographic grade (left-AOR=1.180, $3.800,1.920$; right-AOR=1.190, 3.034, 1.418; $p<0.01$ ).

Conclusions: Large quantities of MSU crystal deposits detected by DECT were found in AxSpA patients without coexisting gout. In addition to AxSpA duration and BASFI score, the MSU crystal deposits at the sacroiliac joint in those patients independently increased the risk of structural joint damage.

Disclosure of Interest: None declared
DOI: 10.1136/annrheumdis-2017-eular.1417

\section{FRI0711 DISCORDANCE OF THE FRAMINGHAM CARDIOVASCULAR RISK SCORE AND THE 2013 AMERICAN COLLEGE OF CARDIOLOGY/AMERICAN HEART ASSOCIATION RISK SCORE IN SYSTEMIC LUPUS ERYTHEMATOSUS AND RHEUMATOID ARTHRITIS}

K. Jafri ${ }^{1}$, A. Ogdie ${ }^{2}$, P. Katz ${ }^{1}$, J. Yazdany ${ }^{1}$, G. Schmajuk ${ }^{1,3} \cdot{ }^{1}$ Rheumatology, University of California, San Francisco, San Francisco; ${ }^{2}$ Rheumatology, University of Pennsylvania, Philadelphia; ${ }^{3}$ Rheumatology, Veterans Affairs Medical Center, San Francisco, United States

Background: Systemic lupus erythematosus (SLE) and rheumatoid arthritis (RA) are associated with an increased risk of cardiovascular (CV) disease, and multipliers to traditional 10-year CV risk scores, such as a EULAR-recommended 1.5 multiplier in RA, have been proposed to capture this increased CV risk. The discordance between CV risk assessment by the Framingham risk score, a modified Framingham risk score (with a 1.5 multiplier), and the more recent 2013 American College of Cardiology/American Heart Association (ACC/AHA) risk score has not been well-studied in patients with rheumatic diseases.

Objectives: To determine the proportion of discordant 10-year Framingham risk scores and 2013 ACC/AHA risk scores in subjects with SLE and RA, both with and without a 1.5 multiplier to the Framingham risk score, and to assess demographic, $\mathrm{CV}$, and rheumatologic clinical characteristics associated with discordant risk scores.

Methods: A cross-sectional study was conducted using SLE and RA subjects drawn from the University of California, San Francisco, Arthritis, Body Composition, and Disability project. 10-year Framingham risk scores, modified Framingham risk scores (with a 1.5 multiplier), and 2013 ACC/AHA risk scores were calculated. As per Adult Treatment Panel-III (ATP-III) recommendations, a subject with a Framingham risk score (or modified Framingham risk score) $\geq 10 \%$ was defined as high-risk by that score, whereas a subject with a Framingham risk score (or modified Framingham risk score) $<10 \%$ was defined as low-risk. A subject with a 2013 ACC/AHA risk score $>7.5 \%$ was defined as high-risk by that score, whereas a subject with a 2013 ACC/AHA risk score $<7.5 \%$ was defined as low-risk. A subject with a discordant risk score was defined as one who had a Framingham risk score (or modified Framingham risk score) that characterized him/her as low-risk and a 2013 ACC/AHA risk score that characterized him/her as high risk. Associations of demographic, $\mathrm{CV}$, and rheumatologic characteristics with discordant risk scores were analyzed using chi-squared tests for categorical variables and using independent $t$-tests for continuous variables.

Results: $11(7.0 \%)$ of the 157 SLE subjects and $11(11.5 \%)$ of the 96 RA subjects had discordant $\mathrm{CV}$ risk scores with low Framingham risk scores but high ACC/AHA risk scores. When the 1.5 multiplier was applied to the Framingham risk score, the number of subjects with discordant risk scores did not significantly change. Rheumatologic disease duration, CRP levels, African-American race, diabetes, current use of anti-hypertensive medication, higher age, and higher systolic blood pressure were all significantly associated with discordant risk scores.

Conclusions: Approximately $10 \%$ of SLE and RA subjects had discordant 10-year $\mathrm{CV}$ risk scores with low Framingham risk scores but high ACC/AHA risk scores, even when a 1.5 multiplier was applied to the Framingham risk score. Prospective studies are needed to address the ability of different CV risk assessment tools, such as the 2013 ACC/AHA risk score, Framingham risk score, and modified risk scores, to predict $\mathrm{CV}$ events in rheumatologic patients, especially those with risk factors associated with discordant risk scores.

Disclosure of Interest: None declared

DOI: 10.1136/annrheumdis-2017-eular.1171

\section{FRI0712 DRIVERS OF UNREFRESHING SLEEP IN PEOPLE WITH MUSCULOSKELETAL PAIN}

K.L. Druce, J. McBeth. Arthritis Research UK Centre for Epidemiology, The University of Manchester, Manchester, United Kingdom

Background: Waking feeling unrefreshed is associated with poor health outcomes including an increased risk of cardiovascular death. Pain is a robust predictor of waking feeling unrefreshed. Pain is a complex disorder and it is not clear whether the pain itself, or associated somatic symptoms, mental health conditions and lifestyle factors, predicts waking unrefreshed.

Objectives: To investigate whether reporting pain was an independent predictor of waking unrefreshed among people with musculoskeletal pain.

Methods: Participants in a population study completed the Estimation of Sleep Problems Scale (ESPS), which indicates the number of days in the past month participants have experienced unrefreshing sleep, problems with sleep onset, maintenance and night awakenings. Pain assessments (body map and duration $>3$ months) were used to classify participants as having no pain, acute pain, chronic pain and CWP (ACR criteria: pain lasting $\geq 3$ months in the axial skeleton and contralateral body quadrants). Participants also reported demographics (date of birth, sex, English Index of Multiple Deprivation); somatic symptoms (Chalder Fatigue Scale (CFQ 11) and Inflammatory Bowel Syndrome (IBS)); mental health (Hospital Anxiety and Depression (HAD) scale); disability (Stanford Health Assessment Questionnaire (HAQ)) and lifestyle factors (average number 